\title{
Decision Support System based Site Quality Evaluation for Plantation
}

\author{
Han Yanyun ${ }^{1}, \mathrm{Wu}$ Baoguo ${ }^{1 *}$ and Wang Chaoqun ${ }^{1}$ \\ ${ }^{1}$ School of Information Science and Technology of Beijing Forestry University, \\ Beijing, China \\ sohocia@sohu.com
}

\begin{abstract}
This paper presents the design and development of a Web-based decision support system for site quality evaluation of plantation (SSQEP). SSQEP is an operat ond automatic evaluation tool that helps farmers in estimating site quality and predicting the growth performance of trees. The SSQEP consists of three subsystems. (i) site quality evaluation for forest land subsystem; (ii) site quality evaluation for non-forest land subsystem; and (iii) stand growth and yield prediction subsystem.

Expert system principle was adopted in the site quality evaluation for non-forest land. An automatic knowledge acquisition module provides a user-friendly interface to domain experts for entering and storing domain-specific knowledge. The knowledge base was used to store and manage the knowledge of domain expents, which is presented as rules and facts. Inference engine was designed and implemented to use forward reasoning mechanism to search the site quality evaluation knowledge.

Furthermore, two site quality evaluation methods for forest land (i.e., site index and growth model methods) were designed in the SSQEP. After evaluating site quality, the stand growth and yield were predicted by a graph.
\end{abstract}

Keywords: site qualif evaluation. decision support system; Knowledge Base; Growth and yield model parse technology

\section{Introduction}

Forest site quality concerns the ability of forest land to grow trees. Thus, site quality evaluation is the foundation of rational land use, which is an effective measure to avoid blind afforestation. Accurate site quality evaluation results can serve as scientific basis for local afforestation and greening. Site quality evaluation is mainly performed by forest experts and experienced farmers on the basis of their experiences. However, a common farmer often encounter d ffficulties in consulting an expert at his or her convenience. Each tree species requires different cropping patterns, and even experienced farmers may not know all relevant information. Therefore, rapid access to all possible information may help farmers make prompt decisions to manage their forest efficiently and effectively. To solve these problems, a computer-aided evaluation system may serve as a powerful tool in speeding up site quality evaluation.

A Web-based decision support system for site quality evaluation of plantation (SSQEP) was designed and developed to inform farmers and grassroots units of forest departments with regard to afforestation and rational use of land resources.

* Corresponding Author 
The rest of this paper is organized as follows: Section 2 presents site quality evaluation methods for forest and non-forest lands. Section 3 introduces the development of the decision support system and discusses all its modules and technologies (i.e., architecture, database, inference engine, growth and yield model parse technology, and user interface). Section 4 concludes this paper.

\section{Site Quality Evaluation Methods}

Site productivity, which is commonly expressed by site index, is influenced by several factors, such as available moisture, climate, and nutrients, along with other soil characteristics, such as soil depth and soil type [1-2]. Site index is the most widely applied measure of site quality in the forestry literature [3-5]. Site index is the average height of dominant trees at a specified base age [6]. The timber production potential of a given site quality is measured by site index [7].

Site quality evaluation methods of plantation was designed, as shown in Figure 1.Two site evaluation methods for forest land were designed in SSQEP, one was site index method and the other was growth model method.

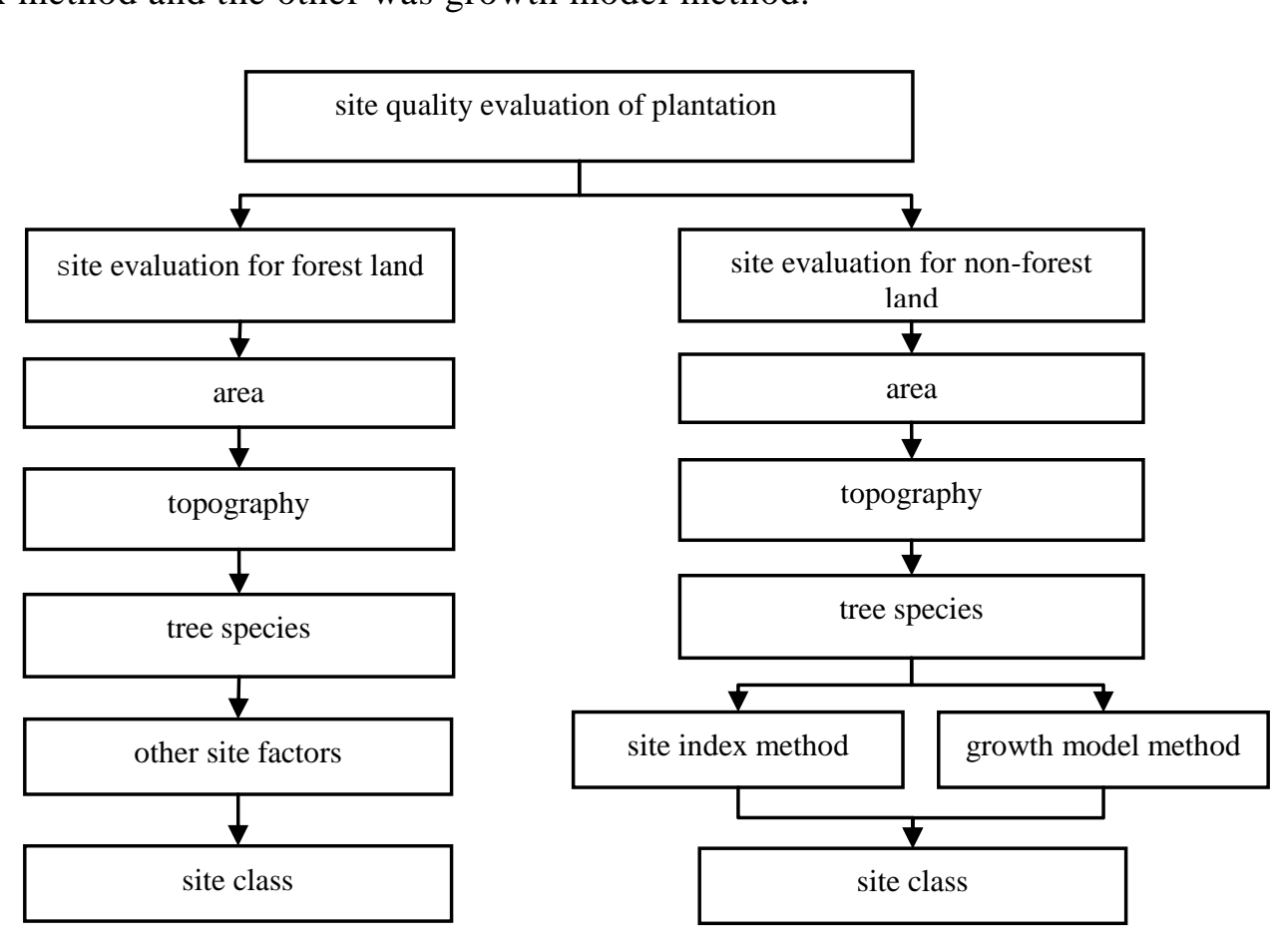

Figure 1. Site Quality Evaluation Method

The site index method obtains the site index based on the mean dominant height and the age of the stand. Thereafter, the system predicts the site class according to the site index. Although site indices have been widely used to predict site conditions, site indices for all kinds of tree species are unavailable in several areas in China. In this case, stand growth model is used to estimate site quality ${ }^{[8]}$. The system retrieves the information that the user entered in the previous Web page and then calculates the mean height of the forest stand based on the stand growth models. The site class is identified according to the height value. 
The expert system method was adopted for site quality evaluation of non-forest land. A user-friendly interface for selecting site conditions, such as area, topography, tree species, and other site factors, was provided. Based on this information, the system provides the site class for non-forest land.

\section{Development of the Decision Support System}

\subsection{Architecture of the System}

The decision support system for the site quality evaluation and artificial forest management has three subsystems: (i) site quality evaluation for forest land subsystem; (ii) site quality evaluation for non-forest land subsystem; and (iii) stand growth and yield prediction subsystem.

Figure 2 shows the architecture of SSQEP. The SSQEP was performed to acquire knowledge related to site quality and stand growth in a user-friendly manner. Site quality evaluation, including site quality evaluations for forest and non-forest lands, provides site classes; such information is considered expert advice. Thereafter, stand growth and yield are predicted by a graph in the stand growth and yield prediction subsystem.

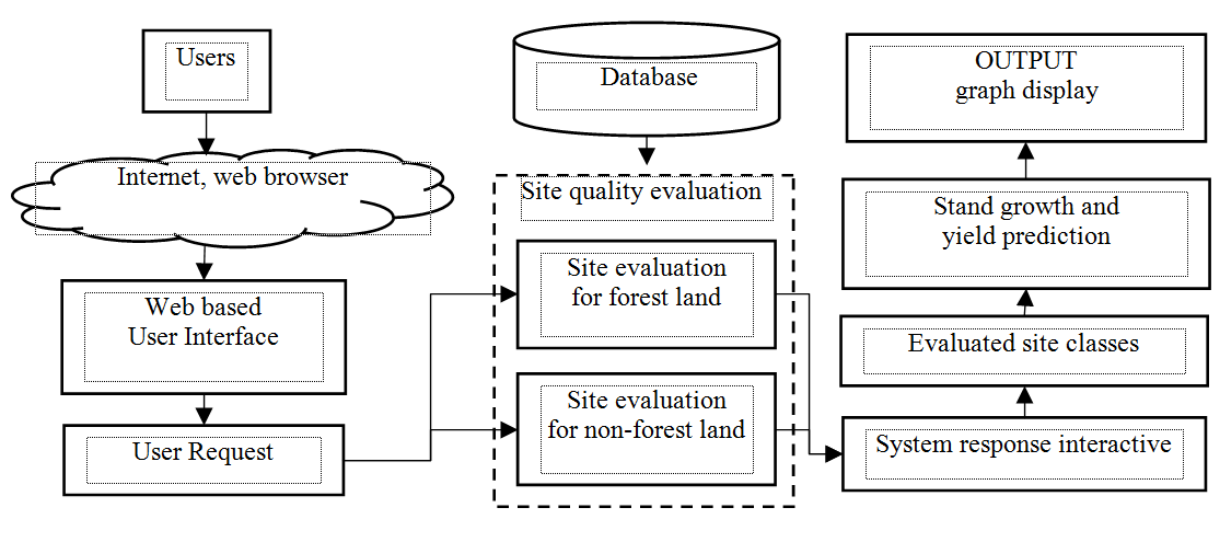

Figure 2. ArChitecture of the Decision Support System

\subsection{Database}

A structured query language server is used to create a relational database for the development of SSQEP. Open database connectivity is used to access the data from the database for site quality evaluation and stand growth and yield prediction. In SSQEP, tables an production rules are used for knowledge representation. All the information of questronnaire for site quality evaluation have been represented in the form of eight database tables (viz. Evaluate_Method, FM_Search, LH_SiteIndex, S_Evaluate, T_GrowthModel, HBLYS_SI, SI_Search and SiteIndex). Seven tables store the facts and data required for the knowledge base, and one table is stored in the model base. Through analysis and iteration of this model, the graph of the stand growth and yield was finally obtained. These tables have been designed in PowerDesigner 12. Figure 3 shows tables' structure and relations among different tables. 

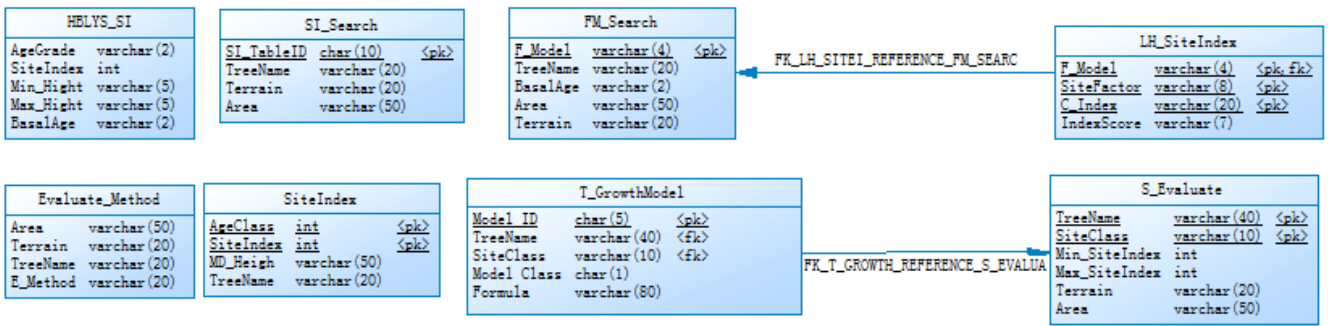

Figure 3. Table Structure and Relations in the Database

3.2.1 Knowledge Base: The knowledge base is used to store and manage the knowledge of SSQEP. The knowledge base contains the domain knowledge required for estimating specific site conditions. The knowledge base is internally represented in tabular form as a relational database that uses an SQL server. The expression of knowledge adopts the production rule.

The following are several examples of rules derived for site quality evaluation of non-forest land:

R1: IF southeast Inner Mongolia AND mountainous topography AND altitude (500 $\mathrm{m}$ to $1000 \mathrm{~m}$ ) AND gentle slope AND lower slope AND brown soil AND larix principis-rupprechtii (aiforestation Species)

THEN the medium site quality

R2: IF southeast Inner Mongolia

AND mountainous topog aphy AND altitude (1000 m to $4500 \mathrm{~m}$ AND steep slope AND sunny slope AND cinnanoon soil AND Chinese pine (afforestation species)

THEN the lower site quality

R3: IF southeast Inner Mongolia

AND mountainous topography

AND altitude $(500 \mathrm{~m}$ to $1000 \mathrm{~m})$

AND lower slope

AND Mongolian pine (afforestation species)

THÊN the superior site quality

R4: IF nor theast Inner Mongolia AND mountainous topography AND sunny slope AND steep slope AND Larix gmelinii(afforestation species)

THEN the medium site quality

R5: IF middle Inner Mongolia AND mountainous topography AND altitude (1000 m to $1500 \mathrm{~m}$ )

AND shady slope AND larix principis-rupprechtii (afforestation species) 
THEN the lower site quality

R6: IF middle Inner Mongolia

AND rolling topography

AND soil type (castano-cinnamon soils)

AND steep slope

AND larix principis-rupprechtii (afforestation species)

THEN the lower site quality

R7: IF south Inner Mongolia

AND rolling topography

AND soil thickness $(30 \mathrm{~cm}$ to $60 \mathrm{~cm})$

AND loessal soil

AND Chinese pine (afforestation species)

THEN the lower site quality

R8: IF western Inner Mongolia

AND soil texture (sticky soil)

AND populus bolleana lauche (afforestation species)

THEN the good site quality

3.2.2. Model Base: The model base is the aggregation of a model with a specific structure ${ }^{[9]}$. The growth and yield models, including stand height-age, stand chameter-age, and stand volume growth models, as a relational database are stored in the model base. Each model is a record that contains the model number, type, and other data items. The structure of the growth and yield model table is shown in Table 1. Store instance is shown in Table 2."

The "Model ID" field is the primary key. The "Site Class" field is categorized into three classes, namely, superior, medium, and low The "Model Class" field is used to identify the model category. The "Formula" field stores the model formula.



The "Model ID" field stores the serial number of the growth and yield models, which is made up of three pats. The following is an example of the serial number of one model:

AAABC

"AAA" 15 coding of tree species, which is in accord with main trees directory of the People's Republic of China. "B" is site class (i.e., "1" represents superior site quality, "20 represents medium site quality, " 3 " represents lower site quality), "C" is model category (i.e., "1" represents the average DBH growth model, "2" represents the average tree height growth model, " 3 " represents the stand volume growth model).

Table 2. MODEL TABLE

\begin{tabular}{|c|c|c|c|c|}
\hline Model ID & Tree Name & Site Class & Model Class & Formula \\
\hline 00812 & fir & superior & $\mathrm{H}$ & $18.29698 *$ power( $1-\exp (-0.103407 * \mathrm{~A}), 1.66829)$ \\
\hline 00822 & fir & medium & $\mathrm{H}$ & $16.658676 *$ power( $1-\exp (-0.07796 * \mathrm{~A}), 1.37458)$ \\
\hline 00832 & fir & lower & $\mathrm{H}$ & $28.0439 *$ power $(1-\exp (-0.023604 * \mathrm{~A}), 1.19559)$ \\
\hline 01311 & arborvitae & superior & $\mathrm{D}$ & $\operatorname{EXP}(3.65512+0.65322 * \operatorname{LOG}(1-\operatorname{EXP}(-0.06 * \mathrm{~A})))$ \\
\hline
\end{tabular}




\begin{tabular}{lllll}
\hline 01321 & arborvitae & medium & D & EXP(3.3073325+0.6880222*LOG(1-EXP(-0.06*A))) \\
01331 & arborvitae & lower & D & EXP $(2.776103+0.804712 * \operatorname{LOG}(1-\operatorname{EXP}(-0.06 * A)))$
\end{tabular}

\subsection{Inference Engine}

Expert system principle was adopted in the site quality evaluation for non-forest land. The site quality evaluation for non-forest land can be broadly divided into four main modules viz., an automatic knowledge acquisition system, knowledge base, inference engine, and a system consultation interface. An automatic knowledge acquisition module provides a user-friendly interface to domain experts for entering and storing domain-specific knowledge. The knowledge base was used to store and manage the knowledge of domain experts, which is presented as rules and facts. Inference engine was designed and implemented to use forward reasoning mechanism to search the site quality evaluation knowledge.

After building the knowledge base, the inference engine was designed and implemented in the SSQEP to use a proper reasoning mechanis $m$ for searching the site quality evaluation knowledge base. It ensures that a proper sequence of questions is asked from the user ${ }^{[10]}$. The system inference engine lses the forward chaining method to guide the search for the selection of questions, rules, and outcomes ${ }^{[11]}$. It matches the knowledge from the knowledge base against the site conditions given by the user and then determines which rules are appropriate.

Figure 4 shows the working process of the inference engine. The inference engine first selects one question from the list of questions entered by the experts for the site quality evaluation for non-forest land. The system displays all the possible answers for the question, and the user selectsone of the answers for each question. The system obtains the input through the user's esponse matches them in the knowledge base, and finds the possible matches (sitelconditions). Based on the user's answers, the system provides the site class evaluation.

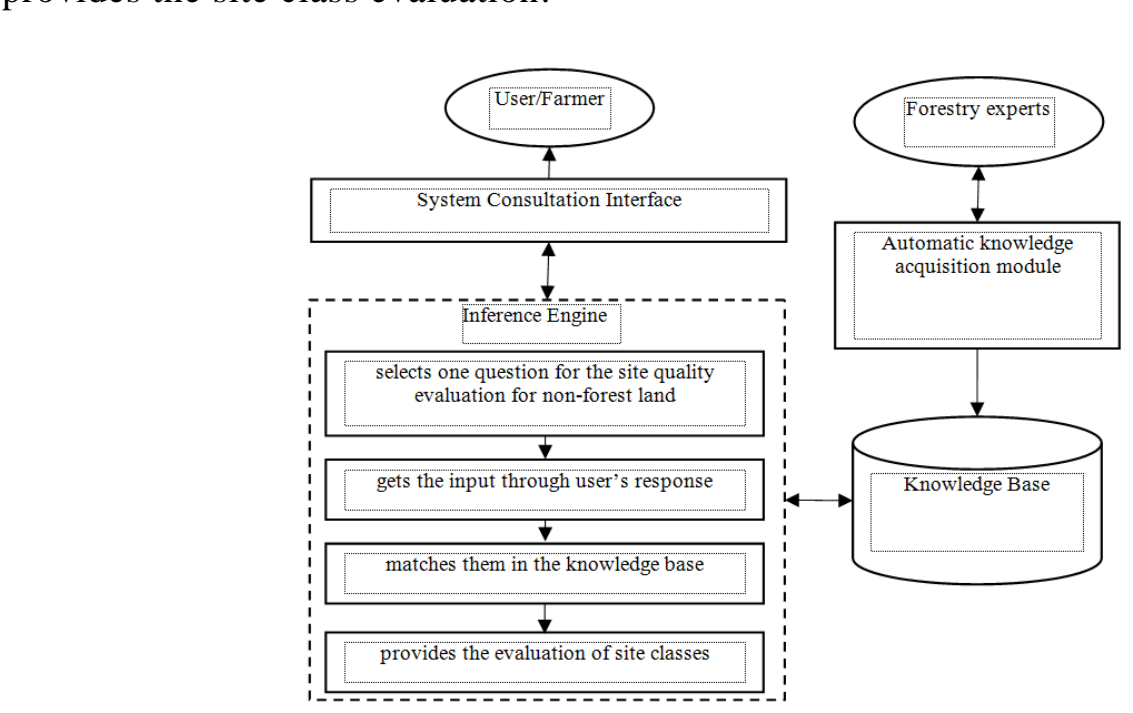

Figure 4. Working Process of the Inference Engine

\subsection{Growth and Yield Model Parse Technology}

The model expression of the growth and yield model was stored in the model base in string format [12]. All the independent variables of the models were presented in 
uppercase letters (e.g., A, D, H, and S signify age, diameter at breast height, tree height, and site quality, respectively), and the mathematical functions are shown in lowercase letters. Model interpreter is usually programmed by high level language, such as $\mathrm{C}++$, C\# or java. Lexical, grammatical and semantic of the formula are analyzed according to the different stand growth models. However, this method's application was complicated [13].

In SSQEP, a more convenient method with structured query language (SQL) was used to interpret and parse the models. Stand information was retrieved from the database with SQL and was then substituted to the independent variables in the formula to compose a set of mathematical expression strings. Finally, the values of the dependent variables (i.e., growth parameters, such as tree height and diameter at breast height) were calculated with an SQL statement.

With Chinese fir used as an example, the formulas of tree height growth forsuperior, medium, and low site qualities were expressed as $18.29698 *$ power (1-exp $(-0.103407 * \mathrm{~A}), 1.66829), 16.658676 *$ power $(1-\exp (-0.07796 * \mathrm{~A}), 1.37458)$, and $28.0439 *$ power $(1-\exp (-0.023604 * \mathrm{~A}), 1.19559)$ respectively [14] The $\mathrm{A}$ in the string expression was replaced by the actual age, which is entered in the previous Web page by the users. Thereafter, the tree height yalues were calculated with the SQL statement according to the formulas. Finally, the site quality class was obtained based on the actual tree height value.

\subsection{User Interface}

Farmers interact with the system any through its lser-friendly interface to obtain results for their queries or to view information. The user operates the system through screens of a graphical user interface. The entire site quality evaluation process is performed in two phases, namely, site class evaluation and stand growth prediction.

Examples of the site quality evaluation for non-forest land are illustrated in Screens 1 (selection of the area), 2 (selection of other site factors, such as elevation, slope, slope direction, slope position, and soil tŷpe), 3 (display of the site evaluation result), and 4 (display of the prediction result of stand growth).

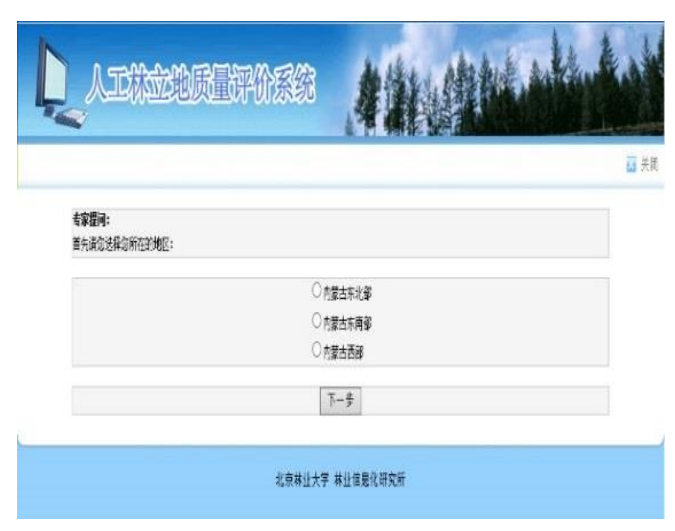

Screen 1. Selection of the Area

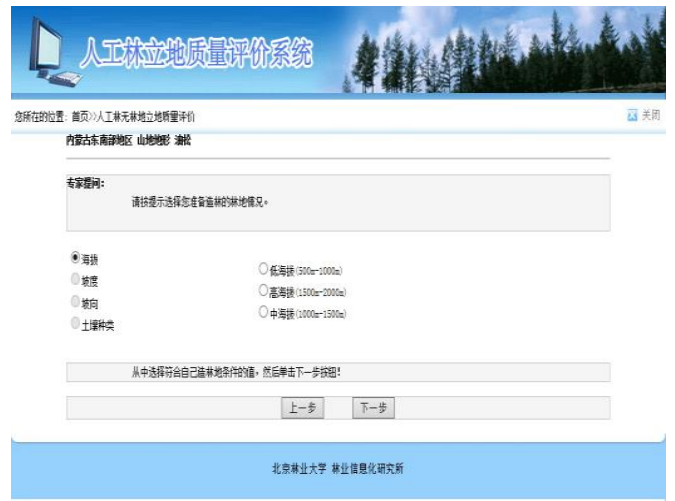

Screen 2. Selection of Other Site Factors 


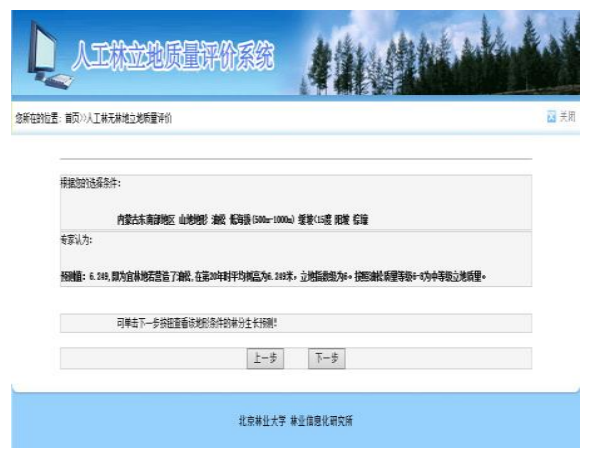

\section{Screen 3. The Site Evaluation Result}
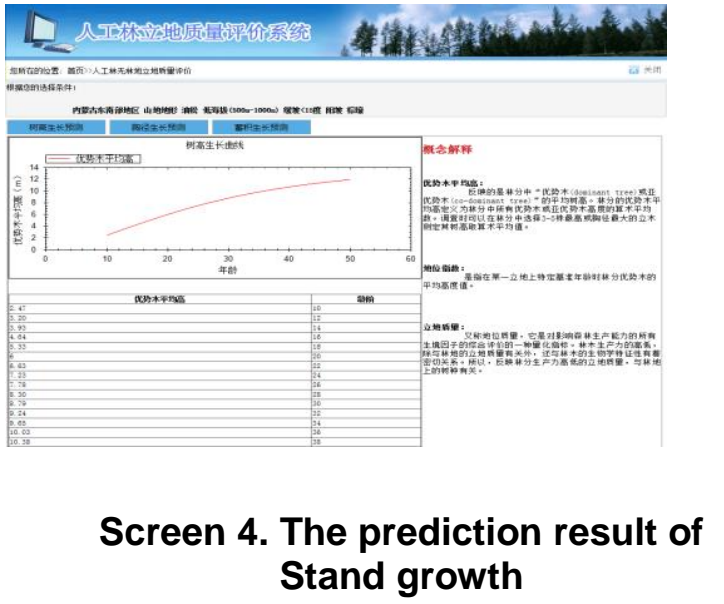

Examples of the site quality evaluation for forest land are illustrated in Screens 5 (selection of the area, terrain and afforestation species), 6 (display of the site evaluation result and the prediction result of stand growth, after inputting stand age, height or diameter of forest stand).

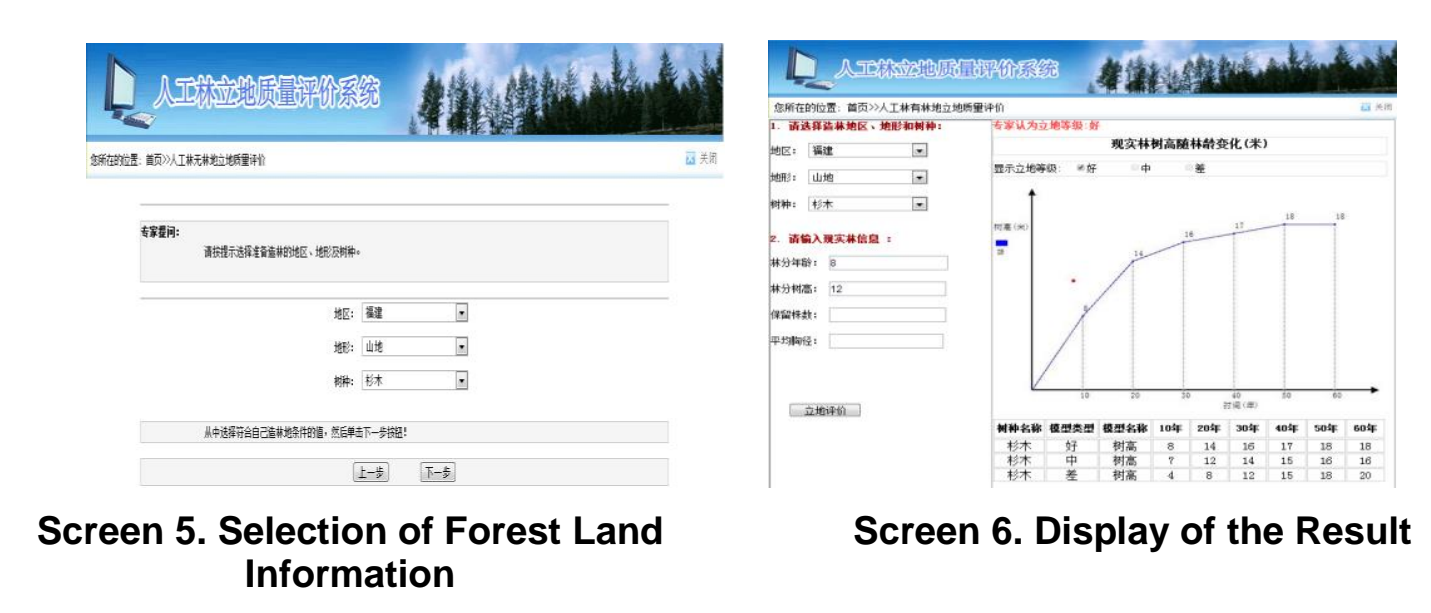

\section{Conclusions}

A Web-based decision support system for site quality evaluation of plantation was designed and developed to provide online help to foresters. The decision support system has three subsystems: (i) site quality evaluation for forest land subsystem; (ii) site quality evaluation for non-forest land subsystem; and (iii) stand growth and yield prediction subsystem.

Site quality evaluation for forest non-land subsystem was designed and developed based on the expert system principle. An automatic knowledge acquisition module was provided to the domain experts for entering and storing domain-specific knowledge. An integrated and consistent knowledge base that combines all the knowledge fed by multiple experts was built in the system. The inference engine of the system uses forward chaining methods that automatically match facts against conditions where rules are applicable. Thereafter, the site class was provided as expert advice. Forest growth and yield are predicted in graph form based on the site class. 
In the site quality evaluation for forest land subsystem, two site quality evaluation methods were used to estimate site class. The system automatically retrieves the site index from the site index table based on the mean dominant height and the age of the stand, which is entered by the users in the system interface. The site class was provided according to the site index. However, the site indices for all kinds of tree species are unavailable in several areas in China. Thus, stand growth models were used to estimate site quality. A model base was designed to store the stand growth and yield models of different site conditions. The model expression of the growth and yield model was stored in the model base in string format. SQL was used to parse the models. Thus, the mean height of the forest stands was calculated. After the site quality was evaluated based on the mean height of the forest stands, the stand growth and yield were predicted by a graph.

\section{Acknowledgements}

This study was supported by the National High Technology Research and Development Program (863 Program) (2012AA102003).

\section{References}

[1] P. Corona, R. Scotti and N. Tarchiani, "Relationship between environmental factors and site index in Douglas-fir plantations in central Italy", Forest Ecology and Management, yol. 110, no. 1 (1998), pp. 195-207.

[2] B. R. Sturtevant and S. W. Seagle, "Comparing estimates of forest site quality in old second-growth oak forests", Forest ecology and management, vol. 191, no. 1, (2004),pp. 311-328.

[3] T. Curt, M. Bouchaud and G. Agrech, "Predicting sitesindex of Douglas-fir plantations from ecological variables in the Massif Central area of France', Forest ecology and management, vol. 149, no. 1, (2001), pp. 61-74.

[4] J. J. Corral Rivas, J. G. Álvarez GonzáleZ, A. D.Rußz González and K. von Gadow, "Compatible height and site index models for five pine species in El Salto, Durango (Mexico)", Forest ecology and management, vol. 201, no. 2, (2004), pp. 145-160.

[5] A. Upadhyay, T. Eid and PL. Sankhayan, "Construction of site index equations for even aged stands of Tectona grandis (teak) from permanent plot data in India", Forest ecology and management, vol. 212, no. 1, (2005), pp. 14-22.

[6] S. Xiaoqian, H. Zhuoqiong, and J. Kabrick "Bayesian spatial prediction of the site index in the study of the Missouri Ozank Forest Ecosystem Project", Computational statistics and data analysis, vol. 52, no. 7, (2008), pp. 3749-3764.

[7] T. Teshome and J. A. Petty, site index equation for Cupressus lusitanica stands in Munessa forest, Ethiopia", Forest ecologx and management, vol. 126, no. 3, (2000), pp. 339-347.

[8] W. Chaoqun and W. Babguo, "Design and implementation of site evaluation system", Computer Engineering and Design, vol. 34, ne. 8, (2013), pp. 2922-2928.

[9] L. Nan, L. Dongming and B. Chunguang, "Research on Development of Corn Production Decision Support System", TELKOMNIKA Indonesian Journal of Electrical Engineering, vol. 11, no. 7, (2013), pp. 37983808.

[10] Devraj and R. Jain, "PulsExpert: An expert system for the diagnosis and control of diseases in pulse crops", Exper Systems with Applications, vol. 38, no. 9 (2011), pp. 11463-11471.

[11] Mansingh, H. Reichgelt and K. M. O Bryson, "CPEST: An expert system for the management of pests and diseases in the Jamaican coffee industry”, Expert systems with Applications, vol. 32, no. 1, (2007), pp. 184192.

[12] H. Zhanguang and W. Baoguo, "Research on database and interpreter of stand growth and yield model", Application Research of Computers, vol. 26, no. 1, (2009), pp. 209-210.

[13] E. Y. Guo, B. G. Wu and Y. Qi, "The Implementation of Stand Growth Visualization Function in Forest Resources MIS", International Journal of Digital Content Technology and its Applications, vol. 6, no. 21, (2012), pp. 605-614.

[14] Z. Yunqing, "Development of experience harvest table of cunninghamia lanceolata plantation in weimin forest farm", Journal of Fujian Forestry Science and Technology, vol. 33, no. 3, (2006), pp. 47-51. 


\section{Authors}

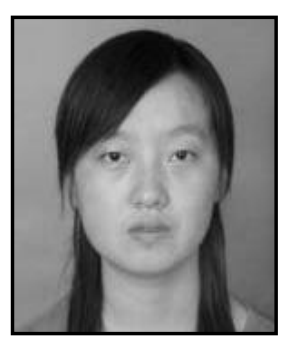

Han Yanyun, she is a Ph.D. candidate in School of Information Science and Technology of Beijing Forestry University, Beijing, China. Her research interests include the forestry information technology, forest resource management and forest decision support system.

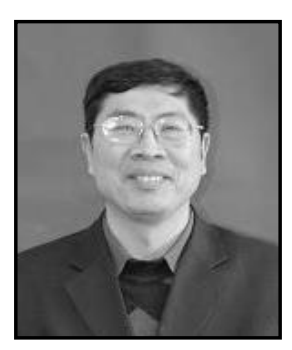

Wu Baoguo, he is currently a professor and $\mathrm{PhD}$ adviser in School of Information Science and Technology of Beijing Forestry University, Beijing, China. His major research focuses on forestry informationization, forestry information technology, forestry expert system decision support system and forest management.

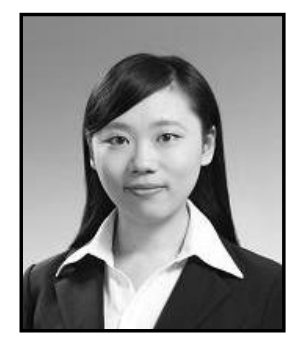

Wang Chaoqun, she received the master's degree from the Beijing Forestry University, in 2013. She is currently working in Beijing JoinCheer Software Co, Ltd.

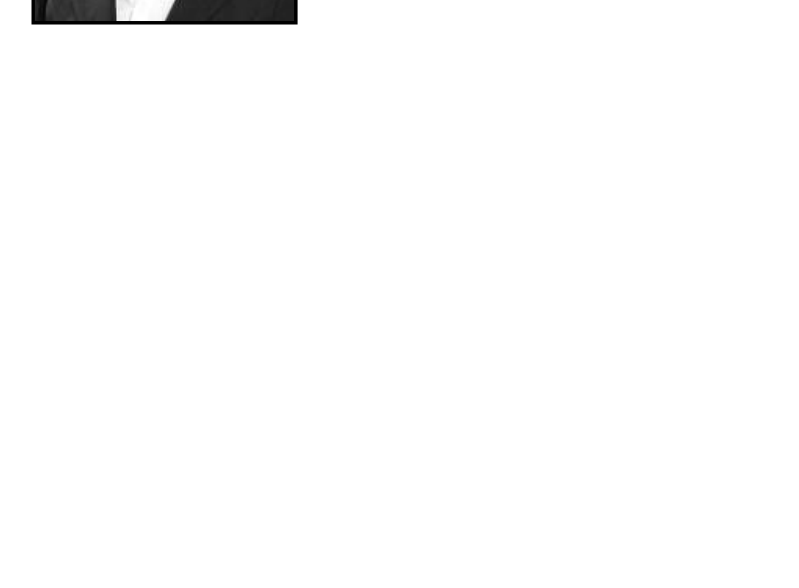

